\title{
Pleomorphic Adenoma of Buccal Mucosa: A Rare Case Report
}

\author{
Ashwini Jangamashetti, BDS ${ }^{1}$, Siddesh Shenoy, MDS $^{2}$, \\ R.Krishna Kumar MDS ${ }^{3}$, Amol Jeur, MS $^{4}$ \\ ${ }^{1}$ Post Graduate Student, Department Of Oral Medicine And Radiology, MARDC,Pune \\ ${ }^{2}$ Reader, Department of oral Medicine and radiology, M.A Rangoonwala Dental College and Research \\ Center, Pune (MARDC), \\ ${ }^{3}$ Professor and HOD, Department of oral Medicine and Radiology, MARDC, Pune \\ ${ }^{4}$ Assistant Professor in Department of General surgery, Krishna Medical College of KIMS Deemed University,
}

\begin{abstract}
Pleomorphic adenoma is a benign tumor of the salivary gland that consists of a combination of epithelial and mesenchymal elements ${ }^{l}$. About $90 \%$ of these tumors occur in the parotid gland and $10 \%$ in the minor salivary glands ${ }^{2}$. Among intra oral pleomorphic adenomas buccal vestibule is among the rarest sites ${ }^{3}$. A case of pleomorphic adenoma of minor salivary glands in the buccal vestibule in a 36 year-old female is discussed ${ }^{4}$. It includes review of literature, clinical features, histopathology, radiological findings and treatment of the tumor, with emphasis on diagnosis ${ }^{4}$. The mass was removed by wide local excision with adequate margins 5 .
\end{abstract}

Keywords: minor salivary gland, pleomorphic adenoma, tumor, parotid gland, vestibule, mesenchymal elements.

\section{Introduction}

Pleomorphic adenoma (PA) is defined by World Health Organization in 1972 as a circumscribed tumor characterized by its pleomorphic or mixed appearance clearly recognizable epithelial tissue being intermingled with tissue of mucoid, myxoid and chondroid appearance ${ }^{2}$. Among all salivary gland tumors, pleomorphic adenoma is the most frequently encountered lesion accounting for approximately $60 \%$ of all salivary gland neoplasms ${ }^{3}$. The palate is considered as the most common Other rare sites include the throat $(2.5 \%)$, retromolar region $(0.7 \%)$, floor of the mouth and the alveolar mucosa ${ }^{5}$.

Pleomorphic adenoma is of glandular origin in the head and neck, usually presenting as a mobile slowly growing, painless firm swelling that does not cause ulceration of the overlying mucosa ${ }^{4}$. PA consists of cells with epithelial and mesenchymal differentiation (mixed tumor) ${ }^{6}$. The morphogenic complexity is because of differentiation of the tumor cells into fibrous, hyalinized, myxoid, chondroid, and even osseous tissue ${ }^{7}$. Patients with pleomorphic adenomas of minor salivary glands usually present in the fourth to sixth decades with a slight predominance in female patients ${ }^{7}$. The buccal vestibule is an uncommon site of occurrence for intraoral pleomorphic adenoma here,we report a case of pleomorphic adenoma in a 36 - year old female patient with characteristic clinical ,imaging, histological features and treatment.

\section{Case report}

A 36 -years -old married female patient (Figure-1) came to the department of oral medicine and radiology with the chief complaint of swelling in the right buccal vestibule of 5 years duration(Figure-2). On clinical examination a swelling of approximate size of $1 \times 2 \mathrm{~cm}$, was noticed and deep proximal caries was seen with 16 (Figure-3.a). On palpation the swelling was firm, and mobile and tooth 16 was non tender . History of presenting illness revealed that the swelling was small in size to start with when noticed by the patient which gradually increased the present size. There was no history of fever, bleeding, pain, pus drainage and loss of sensation. The past dental history and medical history was unremarkable and no other abnormalities were found. The family history was not significant. On general physical examination patient had moderate built, height and well-oriented to time and place with all the vital signs are normal. The patient was adviced for Hematological investigations, IOPA and USG . The hematological report was normal and USG revealed a mass of $16 \times 10 \mathrm{mms}$ with well defined hypoechoic to cystic lesion. Biphasic appearance consisting of epithelial component scattered amidst a chondromyxoid stroma the epithelial cells are made up of cuboidal cells with a rim of cytoplasm and small darker staining myoepithelial cells(Figure-4) .the cells are arranged in groups sheets and interdigitating strands forming small duct like structures at places. The tumour is partly capsulated. A small bit of fibrocollageneous tissue with dense wavy bundles of collagen and covered by hyperplastic stratified squamous epithelium is also seen, There is no evidence of malignancy in the present biopsy. Based upon history, clinical examination, laboratory investigations, ultrasound examination and histopathological findings 
the final diagnosis is benign pleomorphic adenoma of minor salivary glands of right buccal vestibule. To report the recurrence of the lesion the patient is under regular follow up .

\section{Discussion}

Pleomorphic adenomas are benign heterogenous tumors of salivary gland origin. In head and neck region they arise mainly in the major salivary glands mostly parotid $(85 \%)$, submandibular gland $(8 \%)$ and intra oral $(7 \%)$ salivary glands ${ }^{3}$. The palatal glands are the most commonly affected with pleomorphic adenoma with a frequency ranging from $43 \%$ to $70 \%$ followed by the upper lip $(10.1 \%)$ and cheek $(5.5 \%)$. Other rare sites include the throat $(2.5 \%)$, retromolar region $(0.7 \%)$, floor of the mouth, and the alveolar mucosa ${ }^{7}$. This tumor usually presents as a unilateral, painless, slow growing mass in the parotid gland. Few cases are also reported as ulceration, pain, and bleeding ${ }^{7}$.

Minor salivary gland tumors are more common in females than males with a ratio ranging from 1.2:1 to 1.9:1. The age range of patient varies from 9-90 years ${ }^{3}$. The pleomorphic adenomas in youngsters will have similar biological characteristics as in adults but with low recurrence rates after surgical excision ${ }^{7}$. The differential diagnosis of minor salivary gland pleomorphic adenoma of buccal vestibule includes fibroma ,lipoma ,buccal space abscess, foreign body, sebaceous cyst, dermoid cyst, hemangioma , neurofibroma ,rhabdomyoma, adenoid cyst ,carcinoma polymorphous ,low grade adenocarcinoma, The possibility of buccal space abscess was ruled out due to absence of sign of inflammation, hemangioma was ruled out by the absence of any bleeding. Dermoid cyst or lipoma was ruled out as there was no fluctuance. Malignancy was ruled out as these was no induration or fixity to underlying tissues ${ }^{7}$. Absence of punctum and freely movable nature of the mass differentiat Pleomorphic adenomas from sebaceous cyst. As on histological picture both epithelial and myoepithelial cells were seen which rules out mucoepidermoid carcinoma. The negative slip test clinically and absence of lipomatous component histologically rules out lipoma. The absence of perineural invasion and mitotic figures obscure the chance of polymorphic low grade adenocarcinoma.There are three histological subtypes, myxoid (80\% stroma), cellular (myoepithelial cells predominating) and mixed (classic). Pleomorphic adenomas is known to produce recurrence either due to spillage, inadequate removal or enucleation at the time of operation, but is not known to produce distant metastasis ${ }^{2}$. A recurrence rate of $2-44 \%$ has been reported in the literature . the ideal treatment of choice for Pleomorphic adenomas is wide local excision with good safety margins and follow -up of at least 3-4 years ${ }^{2}$.

\section{Conclusion}

pleomorphic adenoma of the buccal vestibul is a rare neoplasm it needs to be diagnosed with a proper history and clinical enuclination complete wide surgical excision is the treatment of choice recurrence after many years of surgical excision as well as malignant transformation should be a concern and therefore long term follow up is necessary ${ }^{7}$.

\section{References}

[1]. M Shruthi, Raghavendra Kini, Vathsala Naik, Girish Y R Naik, Nishith Kumar, Jayanth Kumar. Pleomorphic Adenoma of Palate: A Case Report. International Journal of Advanced Health Sciences. Vol 2 Issue 1• May 2015.

[2]. Pradhuman Verma, Suresh K Sachdeva, Kanika Gupta Verma, Kompal Sachdeva . Pleomorphic adenoma of cheek: A rare case report and review of literature. Indian Journal of Dental Research, 25(1), 2014.

[3]. Preeti Sethi Bakshi, Naveen Jindal, Swati Roy, Kaaveri Khanna. Pleiomorphic Adenoma of Buccal Mucosa: A Rare Case. OHDMVol. 14- No.3-June, 2015.

[4]. Amit Aggarwal, Ravinder Singh,Soheyl Sheikh,Shambulingappa Pallagatti, Isha Singla.Pleomorphic adenoma of minor salivary gland:a case report . RSBO. 2012 Jan-Mar;9(1):97-101 - 99 .

[5]. Taufik Dalati and Mahmoud R Hussein. Juvenile pleomorphic adenoma of the cheek: a case report and review of literature. Diagnostic Pathology 2009, 4:32. Page 2 of 5.

[6]. Ajay Kumar Pillai, Mrinal Satpathy, Saksham Nahar, Swapnil Moghe. Pleomorphic Adenoma in Cheek: An Uncommon Finding . IJSS Case Reports \& Reviews | May-June 2014 | Vol 1 | Issue 1.

[7]. Geetha NT, Deepa BV, Umashankara KV, Rai Kithikumar. Pleomorphic adenoma of minor salivary gland in the cheek. 2015 International Journal of Oral Health Sciences | Published by Wolters Kluwer - Medknow

[8]. Juliana Andrade Cardoso Carla Martins Ferreira,Mariana Moura de Jesus Fernandes Guerra,Gleicy Gabriela Vitória Spinola Carneiro Falcão,Lívia Souza Pugliese,Jener Gonçalves de Farias. Pleomorphic adenoma in the oral mucosa of a young adult: a case report. 290 - RSBO. 2013 Jul-Sep;10(3):289-94 .

[9]. M. Politi , C. Toro, N. Zerman , L. Mariuzzi , M. Robiony . Myoepithelioma of the parotid gland:Case report and review of literature . Oral Oncology EXTRA (2005) 41, 104-108 .

[10]. Simon M.-Y. Lou, Alison M. Rich, Rohana K. De Silva,Martin M.Ferguson . Pleomorphic adenoma of a molar salivary gland . Oral Oncology EXTRA (2006) 42, 170- 172 . 
Figure-1

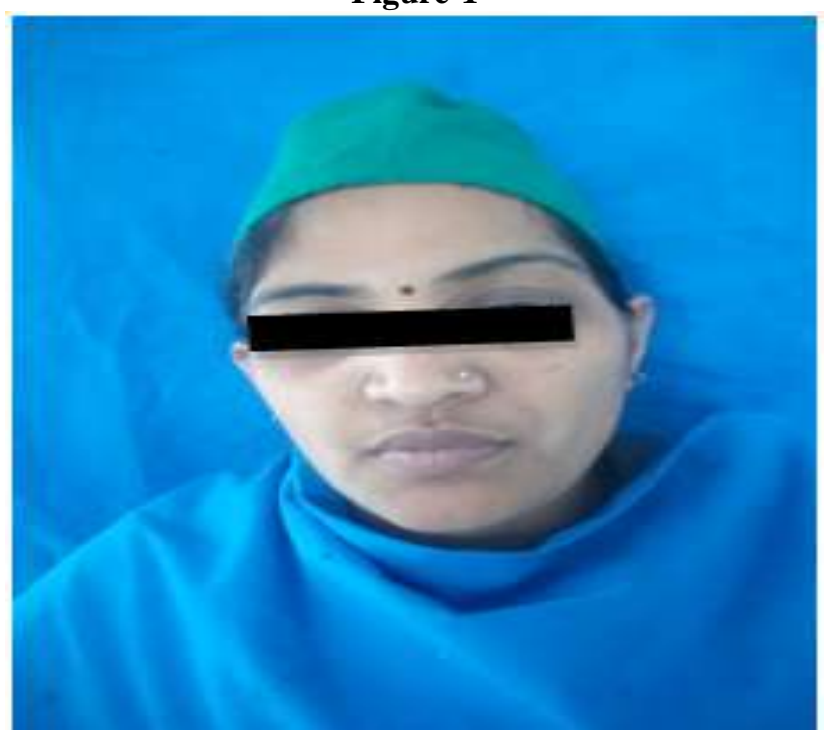

Female Patient

Figure-2

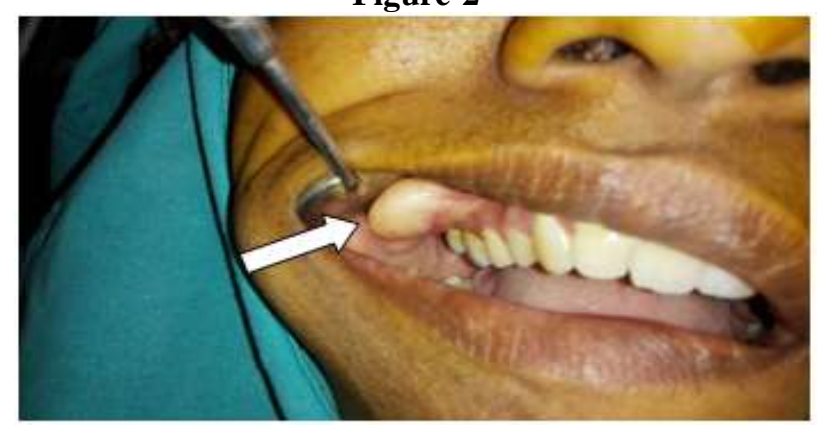

Swelling in the right buccal vestibule

Figure-3

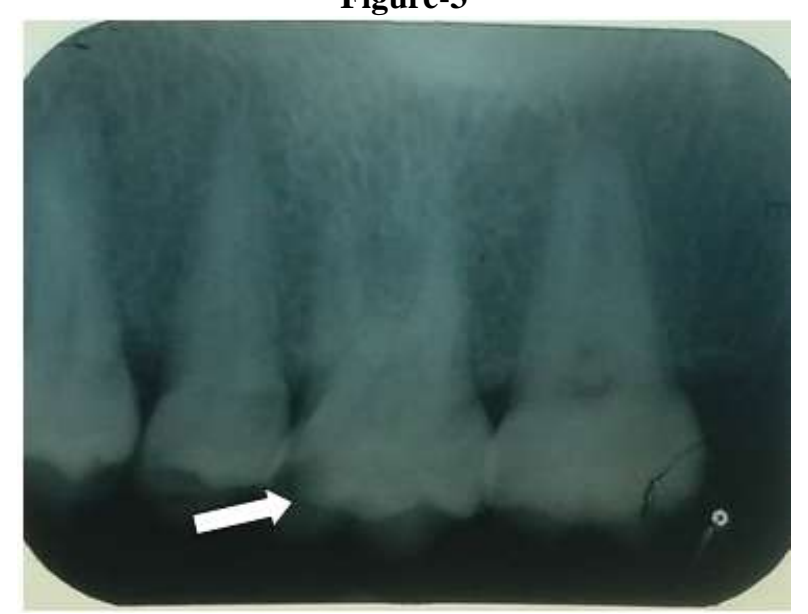

(a). Deep proximal caries 


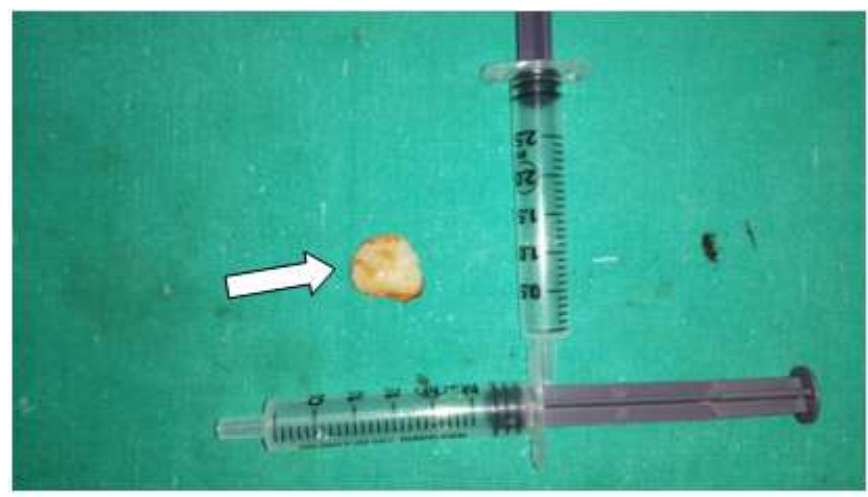

(b). Swelling of approximate size of $1 \times 2 \mathrm{~cm}$

\section{Figure-4}

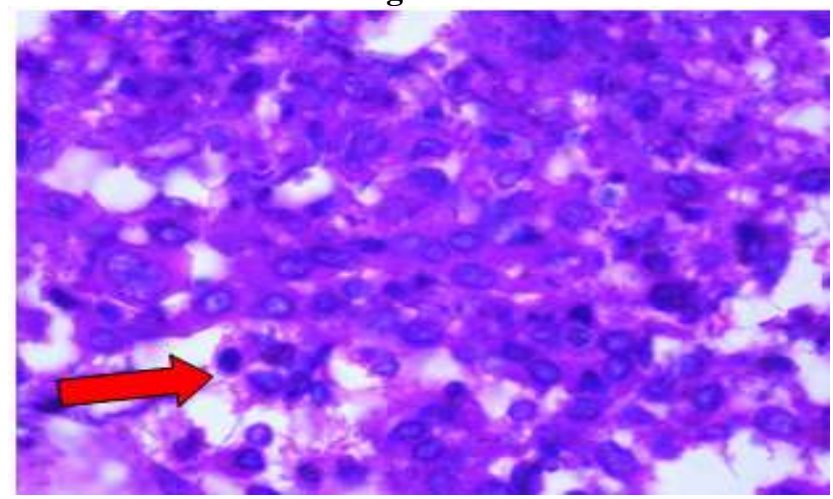

Darker staining myoepithelial cells (red arrow)

Figure-5

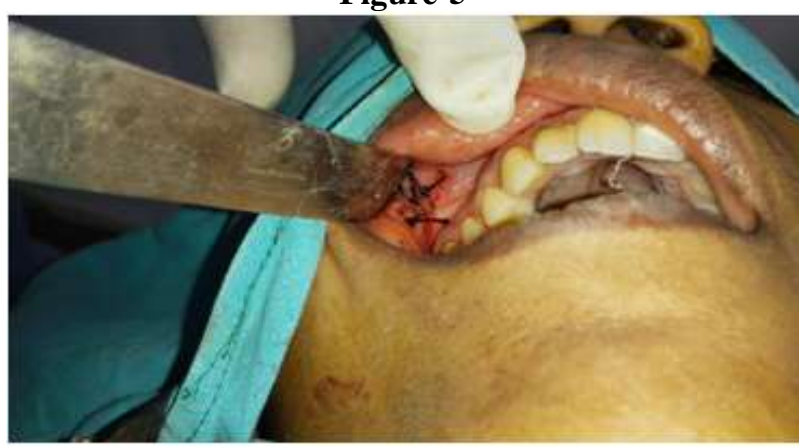

After excision 